\title{
Transition in the Workplace: The Experience of Italian Transgender and Gender Non-conforming People through the Lens of the Minority Stress Theory
}

\author{
CRISTIANO SCANDURRA* \\ cristiano.scandurra@unina.it \\ University of Naples Federico II
}

\author{
VINCENZO BOCHICCHIO \\ vincenzo.bochicchio@unical.it \\ University of Calabria
}

\begin{abstract}
Transgender and gender non-conforming (TGNC) people are a highly stigmatized population experiencing high rates of minority stress and adverse health outcomes. Notwithstanding that, they are able to use adaptive coping strategies to buffer the effect of stigma on their health. Recently, vocational experiences of TGNC individuals were connected to minority stressors, highlighting the effects that stigma have on vocational outcomes. Through the lens of the minority stress theory, this study aimed at exploring the emotional and psychological implications of the gender transition process in the workplace in two maleto-female and six female-to-male Italian TGNC individuals taking part in the study "Reinterpreting organizations through transgender and gender non-conforming narratives". Participants sent their narratives to the editorial board of the PuntOorg International Journal (PIJ). Narratives were analyzed through the thematic inductive analysis. Three macrocategories were identified, each containing core themes, as follows: 1) Minority stressors (a. Transphobia and rejection; b. Felt stigma and stigma anticipation; c. Internalized transphobia; d. Passing as an obstacle); 2) Protective factors (a. Passing as a resource; b. Coming out, visibility, and intimacy with colleagues; c. Resilience); 3) Effect of minority stress on health (a. Depression; b. Anger and suicide ideation). Results and organizational implications are discussed.
\end{abstract}

\section{Introduction}

Transgender and gender non-conforming (TGNC) people are those whose gender identity is not fully aligned with the sex assigned at birth (American Psychological Association, 2015). Due to their gender nonconformity, TGNC people are a highly stigmatized population facing systematic violence and oppression (Bradford et al., 2013) and experiencing high rates of minority stress (Bockting et al., 2013; Hendricks and Testa, 2012; Scandurra et al., 2017b; Testa et al., 2015). Minority stress is a particular form of stress experienced by sexual and gender

\footnotetext{
* Correspondence concerning this paper should be addressed to Cristiano Scandurra, Department of Neurosciences and Reproductive and Odontostomatological Sciences, University of Naples Federico II, Via Pansini 5, Napoli (Italy), 80131. Contact: cristiano.scandurra@unina.it.
} 
minorities due to their stigmatized status (Meyer, 2003, 2007). This theory posits that disparities originate within the stigmatizing social climate to which minority groups are exposed and that, for this reason, these groups are at high risk of developing negative health outcomes. Only recently, scientific literature tried to connect the vocational experiences of sexual and gender minority group to minority stressors, demonstrating that minority stress affects vocational outcomes (e.g., Chung, 2001; Croteau, Anderson and VanderWal, 2008; Ragins, 2008). However, to our knowledge, no previous studies assessed these experiences in Italian TGNC population. Thus, through the lens of the minority stress theory and through a qualitative approach, this study is aimed at understanding the experiences of a group of Italian TGNC people related to the transition in workplaces. Before reporting results, a brief theoretical introduction to the TGNC experience of minority stress will be provided, as well as a brief overview on the workplace experiences of TGNC population.

\section{Minority stress in TGNC population}

Within the context of the individual environmental circumstances, Meyer (2007) conceptualizes distal and proximal stress processes. Distal processes are objective stressors that are independent of the individual because they act without one's own control (i.e., prejudice events, such as experiences of discrimination, violence, and rejection); on the other hand, proximal stressors are dependent on the individual because linked to subjective feelings, thoughts, and actions (i.e., anticipated stigma, or rather concealing one's own sexual orientation and/or gender identity; perceived stigma; and internalized stigma).

Regarding the most distal stressors, the prejudice events, evidence indicates that TGNC people suffer from high levels of violence and discrimination. To this end, Bradford et al. (2013), in a sample of 350 TGNC people recruited in Virginia, reported that $41 \%$ suffered from transgender-related discrimination and that the most associated factors were geographic context, being female-to-male (FtM), low socioeconomic status, belongingness to an ethnic/racial minority group, lack of health insurance, younger age at first transgender awareness, history of violence, substance use, and low levels of family support and community connectedness.

Similarly, there is evidence that psychological problems stem from the stressful living conditions which TGNC people experience. For instance, Bockting et al. (2013), in a sample of 1,093 U.S. TGNC people, reported that social stigma is associated with increased depression, anxiety, and somatization. Shipherd et al. (2011), in a sample of 97 male-to-female (MtF) TGNC people, reported that $98 \%$ suffered from at least one traumatic event and that $91 \%$ suffered from multiple traumatic events; among them, 17.8\% reported post-traumatic stress disorder symptoms, while $64 \%$ reported depressive symptoms.

Compared to distal stressors, less research has been aimed at assessing the impact of proximal stressors on TGNC health. To this end, Beemyn and Rankin (2011) reported that more than half of their sample declared that they hid their gender identity to avoid intimidation. Furthermore, Testa et al. (2012) stated that TGNC people do not report violence to the police and do not have access to medical and mental health services due to the fear of being victimized again. Finally, Bockting et al. (2013) reported higher levels of felt stigma in FtM than in MtF TGNC people, hypothesizing that FtM people pass for biological men easier than MtF for biological women and thus, they cannot experience the benefits of being visible. Instead, 
the most proximal stressor, that is internalized transphobia, can be defined as a discomfort with one's own TGNC identity as a result of internalizing societal negative gender expectations (Bockting, 2015). Perez-Brumer et al. (2015) reported that internalized transphobia increases the likelihood of attempting suicide, while Scandurra et al. (2017b) found a positive association between internalized transphobia and both anxiety and suicide ideation. Furthermore, in a recent study by Scandurra et al. (2018), it was found that internalized transphobia mediates the relationship between enacted stigma and adverse mental health outcomes, and that this relationship is positively buffered by high levels of resilience.

To this end, within the minority stress theory, there is evidence that TGNC individuals use adaptive strategies to buffer the effect of anti-transgender discrimination and internalized transphobia on their health (Pflum et al., 2015; Scandurra et al., 2018; Singh, Hays and Watson, 2011; Singh, Meng and Hansen, 2014; Testa, Jimenez and Rankin, 2014). For instance, Singh, Meng and Hansen (2014) found that community connectedness and social support can reduce the levels of internalized transphobia. In the same vein, Pflum et al. (2015) found that social support can ameliorate the negative mental health outcomes.

\section{Workplace experiences of TGNC people}

Research in the field of workplace experiences of TGNC people is relatively new, although some data are available (e.g., Brewster et al., 2014). For instance, a well-known study by Grant et al. (2011) using a large U.S. sample of 6,450 TGNC individuals, $90 \%$ of participants reported experiencing mistreatment or harassment in their workplaces. Furthermore, $47 \%$ of respondents reported also that, due to their gender nonconformity, they had experienced a negative job outcome, such as being fired, not hired, or denied a promotion. As highlighted by Brewster et al. (2014), these data are alarming considering that TGNC people who experience employment discrimination are approximately five times as likely to experience physical violence (Lombardi et al., 2001).

Contrary to cisgender lesbian, gay, and bisexual (LGB) employees, TGNC people cannot decide to disclose their TGNC identity, especially if they undergo gender transition while performing that job. In a qualitative study by Budge, Tebbe, and Howard (2010), TGNC employees reported being outed by others, fired, denied access to restrooms, and physically threatened. On the same venue, Dispenza et al. (2012) reported experiences of microaggressions. Most of the research on TGNC employees highlighted the negative health outcomes of being discriminated in workplaces, such as stress, anxiety, depression (e.g., Brown et al., 2012; Dispenza et al., 2012), and job dissatisfaction (Brewster et al., 2012).

Notwithstanding these data, several studies have also documented positive experiences related to gender transition in the workplaces. Indeed, based on the minority stress framework, TGNC population is resilient and able to use adaptive coping strategies to face with negative experiences and discrimination (e.g., Scandurra et al., 2017a; Scandurra et al., 2017b; Scandurra et al., 2018). For instance, Singh, Hays and Watson (2011) and Singh, Meng and Hansen (2014) explored resilience within the TGNC population and found that resilience involves both individual traits, such as evolving a self-generated definition of self, embracing self-worth or awareness of oppression, and social characteristics, such as the connectedness to TGNC communities. Both individual and interpersonal traits are effective in decreasing negative outcomes of violence and oppression. Furthermore, some studies specifically 
addressed to TGNC employees documented that a climate resulting TGNC-supportive is associated with greater disclosure of TGNC identity in the workplace, lower job anxiety, and job satisfaction (Brewster et al., 2012; Law et al., 2011). Lastly, in a study by Brewster et al. (2014) analyzing TGNC narratives related to workplace experiences, authors were able to identified both negative experiences (e.g., hostile co-workers, gendered spaces, and the absence of protection policies for employees) and positive strategies for preparing the ground to accept one's own gender transition (e.g., informing human resources or identifying allies).

\section{Aim of the current study}

This study was aimed at exploring the emotional and psychological implications of the gender transition process in the workplace in a small group of Italian TGNC individuals, adopting the lens of the minority stress perspective and letting the "insiders" talk.

\section{Methodology}

\section{Participants and procedures}

Eight TGNC participants (two MtF and six FtM people) took part in the study called "Reinterpreting organizations through transgender and gender nonconforming narratives". The only not Italian participant was removed from the sample because a comparison between an Italian group and one only non-Italian participant was considered scientifically not adequate.

From October 2017 to January 2018, the editorial board of the PuntOorg International Journal (PIJ) launched a "call for narratives" asking TGNC people to "tell personally your own life story related to the world of work, with particular attention (but not exclusively!) to the 'transition in the workplace' issue". The anonymity was guaranteed. All data were collected following the Italian Law on Privacy and Data Protection 196/2003.

\section{Data analysis}

Narratives were analyzed through the thematic inductive analysis (Corbin and Strauss, 2008) which originated from grounded theory. All narratives were treated from an inductive position, in which each interaction of the discourse was considered potentially meaningful (Oberhuber and Krzyżanowski, 2008). This kind of analysis consists of three phases (Corbin and Strauss, 2008). The first phase consists of chunking the data into small units and assigning a code or a descriptor to each unit. The second phase consists of grouping the codes into categories. Finally, the third phase consists of identifying core themes to express the content of each category. All narratives were double-coded by both authors of the current work and the coders discussed possible divergences until an agreement was achieved.

\section{Results}

Through the lens of the minority stress, three macro-categories were identified, each containing core themes, as shown in Table 1. 


\begin{tabular}{ll}
\hline Macro-category & Core themes \\
\hline \multirow{3}{*}{ Minority stressors } & 1. Transphobia and rejection \\
& 2. Felt stigma and stigma anticipation \\
& 3. Internalized transphobia \\
& 4. Passing as an obstacle \\
\hline \multirow{2}{*}{ Protective factors } & 1. Passing as a resource \\
& 2. Coming out, visibility, and intimacy with colleagues \\
& 3. Resilience \\
\hline \multirow{2}{*}{ Effect of minority stress on health } & 1. Depression \\
& 2. Anger and suicide ideation \\
\hline
\end{tabular}

Table 1. Macro-Categories and Core Themes Individuated through the Thematic Inductive Analysis.

Following, each macro-category will be presented separately for clarity reasons.

\title{
Minority stressors
}

Transphobia and rejection theme contains reference to the most distal minority stressor, that is the enacted stigma acted by others towards a TGNC individual. For instance, an FtM participant reported the following words:

\begin{abstract}
I was not exempt from critics but, in hindsight, it was very edifying for me. One day, when I returned from the mess hall, in my group, a friend looked at me, with any kind of kindness, he described me all the embarrassing situations and the loneliness, that I would have experienced, if I had decided to continue to behave like that: I was already old, according to him, I should have stopped, so as not to become ridiculous with a few hairs on my face and a body with an unknown destiny. Taking hormones would have caused unidentified problems to my health, and considering that now everyone obviously already knew me, I would have to stop. He expressed all my fears, I wanted to cry and I could not reply in the same tone
\end{abstract}

As this participant showed, prejudice events have the effect to generate an identity crisis, representing a traumatic experience that affects self-esteem and one's own certainties. These stigmatizing events are visible, objective, and independent of the individual who can only face with them through personal and/or communitarian resources (Hendricks and Testa, 2012; Meyer, 2007), as we shall soon see.

Another impactful minority stressor individuated among narratives has been called felt stigma and stigma anticipation. This minority stressor consists in the expectation that prejudice events will happen and in the subsequent surveillance that requires a high dose of psychic energy (Bockting et al., 2013; Meyer, 2007). For instance, an MtF participant said:

In fact, I couldn't stand my workplace anymore, where I started seeing generalized homotransphobic attitudes. As a consequence, it was so important to say nothing about me above all until my children were ready to handle the pressure created by the news of my 
change. In fact, in my company worked parents whose children went to the same schools and they shared the same interests as mine

We do not know if that workplace was transphobic, but what becomes impactful was the subjective perception of the workplace. As the participant said, she felt the need to hide her TGNC identity to colleagues, and this had a substantial cost in psychological terms.

Again, the most proximal stressor, that corresponds to an identified theme, is the internalized transphobia, or rather an intense discomfort with one's own TGNC identity due to the internalization of society's normative gender expectations (Bockting, 2015; Scandurra et al., 2017b). As an FtM participant expressed, this minority stressor leads to conform oneself to the societal expectations:

As regards the working context, it has not been simple as I work in a gym, that is a quite sexist and narrow-minded work environment. It is also prejudiced against who is not "in accordance" with the idea that society foists on us: the culture of the alpha male and the female who is princess and servant at the same time. [...] I've always been a man and I wanted my place among the men, so sometimes I pushed on the stereotype of the macho man. I felt I had to demonstrate something...

Furthermore, internalized transphobia is strongly associated with negative health outcomes (Scandurra et al., 2017a), as an MtF participant clearly stated:

Nobody knew anything about me and also at work I had to be very careful to keep my real nature secret otherwise it would have provoked a great deal of stress. I started feeling that being always closer and closer to becoming a necessity. At work and at home I had to constrain myself, I didn't want to lose my children, but only during these moments I felt free, I felt myself. I was going crazy

Similarly, another MtF participant shared:

The working situation got harder and harder: I was not feeling comfortable, I felt among enemies. In other words, hell

Finally, the last minority stressor individuated has been called passing as an obstacle. Passing refers to the need to be perceived by others in accordance with one's own experienced gender, in other words, the desire that others perceive them not as TGNC but as cisgender person and, consequently, the concern associated with the difficulty of not being able to pass (Scandurra et al., 2017a). In these terms, the investment in passing for a cisgender person might become a sign of internalized stigma (Bockting, Knudson and Goldberg, 2006) and, thus, distress.

Protective factors 
Passing has a double valence. Indeed, besides being an obstacle, it can also represent a protective factor against the stigma among genderist and heteronormative contexts (Herek and Berril, 1992). In these terms, passing can be an adaptive coping and safety strategy (Hill, 2003), as well explained by two participants, an MtF and an FtM, respectively (passing as a resource):

I truly believe that I was hired with such ease thanks to my 'fitness' for this employment and my 'presentability', I would say

Another identified theme expressing a strong protective factor against the negative effects of stigma on health is coming out, visibility, and intimacy with colleagues. Indeed, despite the initial fear to come out in the workplace, the coming out might have a positive effect on the relationship with colleagues, as it becomes more intimate and personal (Brewster et al., 2012). Resilience also represents a fundamental protective factor against the negative effects of stigma. Indeed, it represents a personal adaptive strategy that TGNC individuals may use to buffer the effects of stress on health, involving the adaptation to risk factors and the capacity to bounce back from adversity (Amodeo et al., 2018; Singh, Hays and Watson, 2011; Singh, Meng and Hansen, 2014), as reported by an FtM participant:

People's words and actions cut like sharp blades and, day by day, they have left in my soul deep wounds that I sometimes feel are open still today, but by now, I know how to heal them, I have become my own doctor.

Furthermore, resilience leads to activate social resources, as it represents also the individual's ability to negotiate with social contexts, generating a greater access to resources. To this end, the words of an FtM participant are enlightening:

After a while, even if every day was new, I was calmer, people felt comfortable with this new colleague, who was a bit strange, with so many new characteristics; some people slowly, others less, some with more practice, all they gradually considered me a man, using masculine form. But I didn't want let my guard down, what I had been able to do, talking about myself, talking about transsexuality, it was too important not only for me, but for every trans person, for that movement in the air that felt like a claim of rights. I had to invent something new. I thought about doing interviews, justifying it as a study I was carrying out. The questions that I invented were: "Do you know transgender people in your circle of friends or acquaintances?" The second one: "What did you think of transsexuality and transgender people until recently?" And the third: "How did the fact that you know me now as a trans person change your idea about me?".

The answers were not all the same and not everyone was ready to talk about it. But I could say that all had the thirst to know more, the empathy towards a new world, the awareness of widespread stereotypes, the importance of every human being, who was searching happiness and self-assertion 


\section{Effect of minority stress on health}

The last macro-category individuated regards the negative effect that minority stress has on health, in particular on mental health (e.g., Bockting et al., 2013; Scandurra et al., 2017b). In this sample, we specifically observed two health outcomes. The first one, depression, has been clearly reported by an MtF participant:

2014 was a very difficult year: stress was high. I was often depressed. I then stayed at home on sick leave. It was on that occasion that the company sent a medical check at home: it turned out I wasn't at home. To be true, during the medical check I was deeply asleep after taking medicine administrated from the psychiatrist of that time. I had to prove everything across psychiatrists' reports that were helping me, with the list of all the prescribed treatments and their effects and, at last, I received a fine anyway. In addition, having a second check-up at the end of the sick week, grew in me the feeling I was an unwelcomed guest. In fact, such incidents happened periodically, above all during periods of sickness, in the previous years

The second one, which is perhaps even more alarming, has been identified as anger and suicide ideation. Through the words of an MtF participant, fired from work:

Every time I walked across the company, I felt bad and I got to the point of imagining to seek revenge on those people responsible for my dismissal. I also thought about ending this forever.

\section{Conclusions and implications}

The current study is limited by the small sample size and its qualitative nature. Notwithstanding that, findings shed light on the role of minority stress processes in workplace experiences of Italian TGNC individuals. Indeed, this study confirms the effectiveness of the minority stress model to read complex phenomena, as that explored in the current work, i.e., "transition in the workplace".

As expected, minority stress processes seem to affect the health of TGNC employees, who are able to resist adopting functional protective factors that protect themselves from the development of negative outcomes. This evidence should lead employers and, in general, work organizations to adopt organizational strategies able to promote those protective factors ameliorating the negative effects of minority stress on health. Indeed, as suggested by Brewster et al. (2014), employers should engage in gender-sensitive practices that would decrease the negativity associated with TGNC transition. For instance, authors suggested to eliminate gender-specific work uniforms, as these uniforms might cause distress to TGNC individuals in transition. In addition, and similarly to the previous good practice, the use of gender-neutral restrooms might alleviate discomfort to TGNC individuals who, on the contrary, could find themselves using the bathroom for people having their same sex assigned at birth, rather than their same actual perceived gender. Again, employers should also provide trainings specifically related to gender transition, helping other co-workers to develop a greater sensitivity and understanding towards TGNG population. 
Thus, we strongly believe that the "diversity management" framework, well described by other scholars participating in the current special issue of PIJ to whom we refer the readers, represents a ground-breaking framework capable to create and promote an inclusive organizational culture within workplaces, as it is aimed at changing the general socioemotional climate in the direction of a more inclusive culture.

\section{Keywords}

minority stress; transgender; resilience; health; transition; workplace; passing; coming out; diversity management.

\section{Reference list}

American Psychological Association (2015) “Guidelines for Psychological Practice with Transgender and Gender Nonconforming People", American Psychologist, 70 (9): 832-864.

Amodeo, A.L., Picariello, S., Valerio, P. and Scandurra, C. (2018) “Empowering Transgender Youths: Promoting Resilience through a Group Training Program", Journal of Gay and Lesbian Mental Health, 22 (1): 3-19.

Beemyn, G., and Rankin, S. (2011) The Lives of Transgender People, New York, NY: Columbia University Press.

Bockting, W.O. (2015) "Internalized Transphobia”, In The International Encyclopedia of Human Sexuality, P. Whelehan and A. Bolin (eds), pp. 583-625, Malden: Wiley-Blackwell.

Bockting, W.O., Knudson, G., Goldberg, J.M. (2006) “Counseling and Mental Health Care for Transgender Adults and Loved Ones", International Journal of Transgenderism, 9 (3-4): 35-82.

Bockting, W.O., Miner, M.H., Swinburne Romine, R.E., Hamilton, A. and Coleman, E. (2013) "Stigma, Mental Health, and Resilience in an Online Sample of the US Transgender Population", American Journal of Public Health, 103 (5): 943-951.

Bradford, J., Reisner, S.L., Honnold, J.A. and Xavier, J. (2013) “Experiences of TransgenderRelated Discrimination and Implications for Health: Results from the Virginia Transgender Health Initiative Study", American Journal of Public Health, 103 (10): 1820-1829.

Brewster, M.E., Velez, B., DeBlaere, C. and Moradi, B. (2012) “Transgender Individuals' Workplace Experiences: The Applicability of Sexual Minority Measures and Models", Journal of Counseling Psychology, 59 (1): 60-70.

Brewster, M.E., Velez, B.L., Mennicke, A. and Tebbe, E. (2014) “Voices from Beyond: A Thematic Content Analysis of Transgender Employees' Workplace Experiences", Psychology of Sexual Orientation and Gender Diversity, 1 (2): 159-169.

Brown, C., Dashjian, L.T., Acosta, T.J., Mueller, C.T., Kizer, B.E. and Trangsrud, H.B. (2012) "The Career Experiences of Male-to-Female Transsexuals", The Counseling Psychologist, 40 (6): 868-894. 
Budge, S.L., Tebbe, E.N., Howard, K.A.S. (2010) “The Work Experiences of Transgender Individuals: Negotiating the Transition and Career Decision-Making Processes", Journal of Counseling Psychology, 57 (4): 377-393.

Chung, Y.B. (2001) "Work Discrimination and Coping Strategies: Conceptual Frameworks for Counseling Lesbian, Gay, and Bisexual Clients", Career Development Quarterly, 50 (1): 3344 .

Corbin, J., Strauss, A. (2008) Basics of Qualitative Research: Techniques and Procedures for Developing Grounded Theory ( $3^{\text {rd }}$ edn.), Thousand Oaks: SAGE.

Croteau, J.M., Anderson, M.Z., VanderWal, B.L. (2008) "Models of Workplace Sexual Identity Disclosure and Management: Reviewing and Extending Concepts", Group $\mathcal{E}$ Organization Management, 33 (5): 532-565.

Dispenza, F., Watson, L.B., Chung, Y.B. and Brack, G. (2012) “Experience of Career-Related Discrimination for Female-to-Male Transgender Persons: A Qualitative Study", The Career Development Quarterly, 60 (1): 65-81.

Grant, J.M., Mottet, L.A., Tanis, J., Harrison, J., Herman, J.L. and Keisling, M. (2011), Injustice at Every Turn: A Report of the National Transgender Discrimination Survey, Washington: National Center for Transgender Equality and National Gay and Lesbian Task Force.

Hendricks, M.L., Testa, R.J. (2012) “A Conceptual Framework for Clinical Work with Transgender and Gender Nonconforming Clients: An Adaptation of the Minority Stress Model", Professional Psychology: Research and Practice, 43 (5): 460-467.

Herek, G.M., and Berrill, K. (1992) Hate Crimes: Confronting Violence against Lesbians and Gay Men. Thousand Oaks: SAGE.

Hill, D.B. (2003) "Genderism, Transphobia, and Gender Bashing: A Framework for Interpreting Anti-Transgender Violence", In Understanding and Dealing with Violence: A Multicultural Approach, B.C. Wallace and R.T. Carter (eds), pp. 113-137, London: SAGE.

Law, C.L., Martinez, L.R., Ruggs, E.N., Hebl, M.R. and Akers, E. (2011) “Trans-Parency in the Workplace: How the Experiences of Transsexual Employees can be Improved", Journal of Vocational Behavior, 79 (3): 710-723.

Lombardi, E.L., Wilchins, R.A., Priesing, D. and Malouf, D. (2001) “Gender Violence: Transgender Experiences with Violence and Discrimination", Journal of Homosexuality, 42 (1): 89-101.

Meyer, I.H. (2003) "Prejudice as Stress: Conceptual and Measurement Problems", American Journal of Public Health, 93 (2): 262-265.

Meyer, I.H. (2007) "Prejudice and Discrimination as Social Stressors", In The Health of Sexual Minorities: Public Health Perspectives on Lesbian, Gay, Bisexual and Transgender Populations, I.H. Meyer and M.E. Northridge (eds), pp. 242-267, New York, NY: Springer.

Oberhuber, F., Krzyżanowski, M. (2008) "Discourse Analysis and Ethnography”, In Qualitative Discourse Analysis in the Social Sciences, R. Wodak and M. Krzyżanowski (eds), pp. 182203, Basingstoke: Palgrave Macmillan. 
Perez-Brumer, A., Hatzenbuehler, M.L., Oldenburg, C.L. and Bockting, W.O. (2015) “Individual- and Structural-Level Risk Factors for Suicide Attempts among Transgender Adults", Behavioral Medicine, 41 (3): 164-171.

Pflum, S.R., Testa, R.J., Balsam, K.F., Goldblum, P.B. and Bongar, B. (2015) "Social Support, Trans Community Connectedness, and Mental Health Symptoms among Transgender and Gender Nonconforming Adults", Psychology of Sexual Orientation and Gender Identity, 2 (3): 281-286.

Ragins, B.R. (2008) “Disclosure Disconnects: Antecedents and Consequences of Disclosing Invisible Stigmas Across Life Domains", The Academy of Management Review, 33 (1): 194215.

Scandurra, C., Amodeo, A.L., Bochicchio, V., Valerio, P. and Frost, D.M. (2017a) “Psychometric Characteristics of the Transgender Identity Survey in an Italian Sample: A Measure to Assess Positive and Negative Feelings towards Transgender Identity", International Journal of Transgenderism, 18 (1): 53-65.

Scandurra, C., Amodeo, A.L., Valerio, P., Bochicchio, V. and Frost, D.M. (2017b), “Minority Stress, Resilience, and Mental Health: A Study of Italian Transgender People", Journal of Social Issues, 73 (3): 563-585.

Scandurra, C., Bochicchio, V., Amodeo, A.L., Esposito, C., Valerio, P., Maldonato, N.M., Bacchini, D. and Vitelli, R. (2018), "Internalized Transphobia, Resilience, and Mental Health: Applying the Psychological Mediation Framework to Italian Transgender Individuals", International Journal of Environmental Research and Public Health, 15 (3): E508.

Shipherd, J.C., Maguen, S., Skidmore, W.C. and Abramovitz, S.M. (2011) "Potentially Traumatic Events in a Transgender Sample: Frequency and Associated Symptoms", Traumatology, 17 (2): 56-67.

Singh, A.A., Hays, D.G., Watson, L.S. (2011) "Strength in the Face of Adversity: Resilience Strategies of Transgender Individuals", Journal of Counseling and Development, 89 (1): 2027.

Singh, A.A., Meng, S.E., Hansen, A.W. (2014) “'I Am My Own Gender': Resilience Strategies of Trans Youth", Journal of Counseling and Development, 92 (2): 208-218.

Testa, R.J., Habarth, J., Peta, J., Balsam, J. and Bockting, W.O. (2015) “Development of the Gender Minority Stress and Resilience Measure", Psychology of Sexual Orientation and Gender Identity, 2 (1): 65-77.

Testa, R.J., Jimenez, C.L., Rankin, S. (2014) “Risk and Resilience during Transgender Identity Development: The Effects of Awareness and Engagement with Other Transgender People on Affect", Journal of Gay and Lesbian Mental Health, 18 (1): 31-46.

Testa, R.J., Sciacca, L.M., Wang, F., Hendricks, M.L., Goldblum, P., Bradford, J. and Bongar, B. (2012) "Effects of Violence on Transgender People", Professional Psychology: Research and Practice, 43 (5): 452-459. 